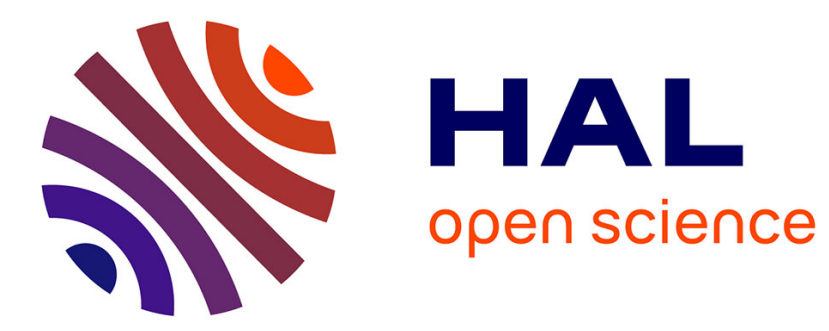

\title{
pGOODs: new plasmids for the co-expression of proteins in
}

Emanuele Conte, Gianpiero Landolfi, Gabriele Vincelli, Alessandra Stefan, Alejandro Hochkoeppler

\section{- To cite this version:}

Emanuele Conte, Gianpiero Landolfi, Gabriele Vincelli, Alessandra Stefan, Alejandro Hochkoeppler. pGOODs: new plasmids for the co-expression of proteins in. Biotechnology Letters, 2011, 33 (9), pp.1815-1821. 10.1007/s10529-011-0624-6 . hal-00690283

\section{HAL Id: hal-00690283 \\ https://hal.science/hal-00690283}

Submitted on 23 Apr 2012

HAL is a multi-disciplinary open access archive for the deposit and dissemination of scientific research documents, whether they are published or not. The documents may come from teaching and research institutions in France or abroad, or from public or private research centers.
L'archive ouverte pluridisciplinaire HAL, est destinée au dépôt et à la diffusion de documents scientifiques de niveau recherche, publiés ou non, émanant des établissements d'enseignement et de recherche français ou étrangers, des laboratoires publics ou privés. 
pG0ODs: new plasmids for the co-expression of proteins in Escherichia coli

Emanuele Conte*, Gianpiero Landolfi*, Gabriele Vincelli*, Alessandra Stefan*o, Alejandro Hochkoeppler*\$

* Department of Industrial Chemistry, University of Bologna, Viale Risorgimento 4, 40136 Bologna (I)

${ }^{\circ}$ CSGI, Department of Chemistry, University of Florence, Via della Lastruccia 3, 50019 Sesto Fiorentino (I)

\$To whom correspondence should be addressed:

Prof. Alejandro Hochkoeppler

Department of Industrial Chemistry

University of Bologna

Viale Risorgimento 4

40136 Bologna (Italy)

Tel.: ++ 390512093671

Fax: ++ 390512093673

E-mail: a.hochkoeppler@unibo.it

Key words: ColE1 origin; compatible plasmid; Escherichia coli; expression vector; p15A origin. 


\begin{abstract}
Two systems for the co-expression of proteins in Escherichia coli were designed and constructed. The first system relies on the new vector, pGOOD, which is compatible with ColE1-type plasmids and sustains efficient co-expression of soluble protein complexes. The second system is based on the pGOOD1 vector (a derivative of pGOOD), useful for the production of toxic proteins, whose synthesis can be regulated by the co-expressed LacI repressor.
\end{abstract}

\title{
Introduction
}

The study of protein-protein interactions is a major challenge in modern biochemistry and molecular biology, and important efforts have been made to design and construction of tools for the rapid identification of interacting proteins. An important advance in this field was the introduction of the yeast two-hybrid system (Fields and Song, 1989). More recently, bacterial two-hybrid systems have also been developed (Karimova et al., 1998; Joung et al., 2000). Both methodologies are suitable for extensive screenings of interactors. Generally, two-hybrid systems rely on the use of fusion proteins: the proteins to be tested are fused, generating hybrids, to appropriate reporters of the intervening interaction. Unfortunately, this approach cannot be applied to those proteins (e.g. of small molecular mass) whose folding is significantly altered by the fusion with a reporter. Other powerful techniques relying on protein reporters, such as FRET (Stryer and Haugland, 1967; Ha et al., 1996), suffer from 
the same limitations. A possible solution to this drawback is the co-expression of native proteins, whose interaction has yet to be tested and studied in detail. Moreover, protein-DNA interactions can also be studied by co-transforming a bacterial host with: i) a plasmid bearing the target DNA; ii) a vector suitable for the expression in vivo of the protein of interest. This method was reported in an elegant study by Müller-Hill, who dissected the interaction of lac repressor with operators (Lehming et al., 1987). In recent years, different co-expression vectors have been constructed and described (Zhang et al., 2007; Walia et al., 2007; Zeng et al., 2010). These vectors are useful because they are compatible with the most commonly used expression plasmids for Escherichia coli, containing a ColE1type origin of replication (ori). In particular, the pEC plasmid (Zhang et al., 2007) contains the pEIB1 ori, an L-arabinose-inducible promoter, and a kanamycinresistance cassette. This vector is a stable and high-copy number plasmid in $E$. coli. The pCR2 series comprises plasmids containing the pCR2 ori from Corynebacterium renale, the lac or the $\mathrm{T} 7$ promoter, and an ampicillin- or a chloramphenicol-resistance cassette (Walia et al., 2007). These vectors were reported as high-copy number and were successfully used for the expression in E. coli of GFP or human interferon- $\gamma$. The pHEX plasmid contains the p15A ori, the T7 promoter, and a kanamycin-resistance cassette (Zeng et al., 2010). This vector was successfully used to co-express two proteins from Mycobacterium tubercolosis $\mathrm{H} 37 \mathrm{Rv}$ in E. coli. While useful for co-expression experiments, these plasmids do not provide either a low-copy number expression system, or a 
multiple-cloning-site (MCS) identical to one already available in another plasmid. Importantly, a co-expression vector featuring both these properties would allow: i) the easy shuttling of clones between low- and high-copy number expression vectors sharing the same MCS; ii) the co-expression of two proteins, one in excess of the other, and vice versa. To take advantage of these features, we have constructed the pGOOD expression vector, featuring the p15A ori, a tetracycline-resistance cassette, the lac repressor-promoter-operator regulatory elements, and the MCS from pTrcHisB. This MCS is identical to the one present in the pBADHis series, making the entry of two clones into pBADHis and into pGOOD easily reversable. In addition, we have constructed two additional plasmids, i.e. pGOOD1 and pGOOD2, both devoid of the lacI gene, and respectively containing the $O_{i d}$ and the $O_{3}$ lac operator. The expression of genes hosted in these plasmids can be tightly controlled by the lac repressor produced from a different vector, e.g. pBADHis.

This paper describes the co-expression of proteins in E. coli using pGOODs and pBADHisB, along with the stability features of the new plasmids.

\section{Materials and methods}

\section{Bacterial strains, plasmids and growth media}

All the experiments were performed using Escherichia coli TOP10 (genotype: F-, mcrA, $\triangle(m r r-h s d M R S-m c r B C), \Delta l a c X 74, \phi 80 l a c Z 4 M 15$, deoR, endA1, recA1, araD139, $\Delta$ (araA-leu)7697, galU, galK, rpsL, nupG). The plasmids pTrcHisB and 
pBADHisB were obtained from Invitrogen (Carlsbad, USA). The pACYC184 vector was a generous gift of New England Biolabs (Ipswich, USA). The pBAD$\alpha 1160$ and pBAD-ع243 plasmids were previously described (Bressanin et al., 2009). The DNA fragment coding for $\varepsilon 243$ was excised from pBAD (using NcoI and PstI enzymes) and inserted into pGOOD (NcoI and PstI sites), yielding pGOOD- $\varepsilon 243$. Bacteria were grown, unless specified, at $37^{\circ} \mathrm{C}$ using LB medium (tryptone, yeast extract, and $\mathrm{NaCl}$ at 10, 5, and $10 \mathrm{~g} / \mathrm{l}$, respectively). The growth medium was supplemented, when appropriate, with ampicillin and/or tetracycline at 100 and $10 \mu \mathrm{g} / \mathrm{ml}$, respectively.

\section{DNA manipulation}

DNA restriction, ligation, and transformation of E. coli were performed according to standard procedures (Sambrook and Russell, 2001). The partial digestion of pTrcHisB with SspI was performed at $37{ }^{\circ} \mathrm{C}$ for $4 \mathrm{~h}$ using an enzyme concentration 20 times lower than that recommended by the manufacturer for standard restrictions. In addition, the SspI enzyme was used in a sub-optimal reaction buffer $(20 \mathrm{mM}$ Tris/acetate, $10 \mathrm{mM}$ magnesium acetate, $50 \mathrm{mM}$ potassium acetate, $1 \mathrm{mM}$ dithiothreitol, $\mathrm{pH}$ 7.9), in the presence of which activity is $50 \%$ when compared to the maximal value.

\section{Co-expression experiments}

E. coli TOP10 was transformed with the pBAD- $\alpha 1160$ vector. Transformants were selected using LB/ampicillin medium. They were then purified and used to obtain electrocompetent cells into which the pGOOD-ع243 plasmid was 
introduced. Overnight cultures of co-transformants (selected and purified with LB-ampicillin-tetracycline medium) were diluted (1:500) into fresh medium. At late logarithmic phase (5 h after dilution of pre-cultures) inducer(s) were added to the culture medium and cells harvested $3 \mathrm{~h}$ after inducer(s) addition. Total protein extracts were obtained by sonication and the soluble fraction isolated by centrifugation $(16,000 \mathrm{~g}, 20 \mathrm{~min})$. Gel filtration was performed with a $1.6 \times 70 \mathrm{~cm}$ Superdex 200 column, calibrated with HMW and LMW Gel Filtration Calibration kits (GE Healthcare). According to the calibration, fractions 6, 42, and 52 contained proteins featuring molecular mass equal to 819,52 and $24 \mathrm{kDa}$, respectively. The pGOOD- $\varepsilon 243-\theta$ plasmid was obtained by inserting (using PstI and EcoRI sites) into pGOOD-ع243 a synthetic gene containing the araB-araA intergenic region and the holE gene. Gene synthesis was performed by Entelechon GmbH (Regensburg, Germany).

\section{Stability of pGOOD in vivo}

To assess the stability of pGOOD in E. coli TOP10, pre-cultures grown in selective medium (LB supplemented with tetracycline) were diluted in LB devoid of tetracycline and propagated for 30 generations. At intervals corresponding to 10 generations, aliquots of the cultures were withdrawn, diluted and spread onto LB and LB+tetracycline medium. The fraction of tetracycline-resistant individuals was then determined. Each aliquot of the cultures was also used to extract and purify pGOOD; after gel electrophoresis, the amount of pGOOD 
recovered from each sample was estimated with Quantity One software (BioRad, Hercules, USA).

\section{Assay for mutator phenotype}

E. coli TOP10 was co-transformed with pGOOD1 containing an insert (NcoI and PstI sites) coding for the D12A variant of DNA Pol-III $\varepsilon$ subunit. Mutation of the catalytic residue D12 should abolish the proofreading activity of this subunit (Fijalkowska and Schaaper, 1996). E.coli TOP10/pG00D1-عD12A was then cotransformed with pBAD containing (between NcoI and Pst sites) the lacI gene or a lacI $^{\text {ts }}$ mutant previously described by Bukrinsky et al. (1988). Cotransformants were pre-cultured at $30^{\circ} \mathrm{C}$ in LB medium supplemented with 13 $\mathrm{mM}$ arabinose; pre-cultures were then diluted (1:500) in fresh arabinosecontaining $\mathrm{LB}$ medium and grown at $30^{\circ} \mathrm{C}$ for about 10 generations. Finally, the cultures were diluted (1:500) in fresh arabinose-free LB medium and grown at $42{ }^{\circ} \mathrm{C}$ for a further 10 generations. All growth media were used devoid of IPTG. The frequency of mutant individuals was assayed by spreading aliquots of the cultures (grown at 30 or at $42{ }^{\circ} \mathrm{C}$ ) onto LB-agar Petri dishes supplemented or not with kanamycin $(30 \mu \mathrm{g} / \mathrm{ml})$. It is important to note that resistance to kanamycin can be a more sensitive phenotype than rifampicin-resistance (Monteiro et al., 2003). The frequency of mutants was determined by considering three independent cultures of each sample. 


\section{Results and discussion}

To construct pGO0D, the pACYC184 vector was subjected to total digestion with BstZ17I and MscI restriction enzymes (Fig. 1), yielding three DNA fragments of 2345 (BstZ17I-MscI), 1042 (MscI-MscI) and 858 (MscI-BstZ17I) bp, respectively (Fig. 1). The digested DNA was electrophoresed and the $2345 \mathrm{bp}$ fragment containing the p15A ori and the tetracycline-resistance marker was recovered and purified (Fig. 1). The pTrcHisB vector was in parallel subjected to total digestion with BStZ17I and AlwNI restriction enzymes, and the reaction products were further treated with SspI enzyme. In this case, a partial digestion with SspI was performed to recover a $2480 \mathrm{bp}$ fragment containing the lac repressor, promoter-operator, the MCS and the transcriptional terminators of pTrcHisB (Fig. 1). This fragment was purified by gel electrophoresis, and then ligated to the BstZ17I-MscI fragment of pACYC184, yielding the pG00D vector (Fig. 1). Due to their presence in the BstZ17I-MscI fragment of pACYC184 the restriction sites recognized by BamHI, BstBI, HindIII, and NheI are not uniquely located in the MCS of the pTrcHisB portion of pGOOD. However, the MCS of pGOOD does contain the following unique sites: NcoI, BsaBI, SacI, Eco53kI, TliL, Paer7I, XhoI, BglII, PstI, KpnI, Acc65I, NdeI, and EcoRI. With the exception of BsaBI and NdeI, these sites are also present in the MCS of pBADHisB, facilitating gene shuttling between this plasmid and pGOOD.

The stability of the new plasmid was tested in Escherichia coli TOP10: pGOOD was introduced into this strain, the transformants were selected and pre- 
cultured in the presence of tetracycline, and then cultured in antibiotic-free LB medium for 30 generations. We did not observe a significant loss of plasmid during this time; remarkably, after 30 generations $100 \%$ of individuals were still tetracycline-resistant and the yield of plasmid recovery was not significantly affected by propagation in host cells (data not shown).

These observations indicate a good maintenance of pGOOD, especially when considering that the plasmids containing the p15A ori are known as low-copy number vectors in E. coli. Moreover, pACYC184 was reported as an unstable plasmid when the host strain is cultivated in minimal medium supplemented with a limiting amount of glucose (Lenski and Bouma, 1987). Notably, these previous findings suggest an easy procedure for the cure of pGOOD from host cells, i.e. the shift of transformant cultures from rich to minimal medium. The performance of pGOOD as a co-expression vector was tested by co-transforming E. coli TOP10 with: i) the pBAD- $\alpha 1160$ plasmid (Bressanin et al., 2009), which contains an insert coding for the $\alpha$ (polymerase) subunit of E. coli DNA polymerase III (DNA Pol-III); ii) the pGOOD vector bearing the gene coding for the $\varepsilon$ (proofreading) subunit of DNA Pol-III. Co-transformants were grown in the presence of both ampicillin and tetracycline, and induced for overexpression of $\alpha$ or $\varepsilon$ by addition to the culture medium of arabinose or IPTG, respectively. In addition, control cultures were grown in the absence of any inducer. When only arabinose was used, overexpression of DNA Pol-III $\alpha$ subunit was observed, while in the absence of inducer no appreciable expression from pBAD of this 
protein was detectable (Fig. 2A, cf. lanes 1 and 2). This observation agrees with the regulatory properties of the pBAD vectors, which were described as tightlyregulated expression tools (Guzman et al., 1995). When only IPTG was used, overexpression of the DNA Pol-III $\varepsilon$ subunit was detected (Fig. 2A, lane 3). In the absence of inducer, a limited but significant expression of $\varepsilon$ was observed (Fig. 2A, lane 1). The expression of $\varepsilon$ from pGOOD in the absence of IPTG was confirmed by western blotting (data not shown), using a previously described rabbit polyclonal anti- $\varepsilon$ antibody (Bressanin et al., 2009). Again, this finding is in line with previous reports demonstrating that the basal expression level of cloned genes is higher in lac-based expression vectors than in pBAD plasmids (Guzman et al., 1995).

When the growth medium was supplemented with both arabinose and IPTG, a consistent overexpression of DNA Pol-III $\alpha$ and $\varepsilon$ subunits was observed (Fig. 2A, lane 4), indicating that the pBAD-pGOOD vector couple can be efficiently used to co-express proteins in E. coli. Moreover, we were able to modulate the levels of co-expressed $\alpha$ and $\varepsilon$; when IPTG was added 30 min before arabinose, the concentration of $\varepsilon$ increased at the expense of the overexpression of $\alpha$ (data not shown). To test the feasibility of co-expression of a ternary protein complex, the holE gene, coding for the $\theta$ subunit of DNA Pol-III, was inserted into pGOOD$\varepsilon 243$ (using the PstI and EcoRI sites) and the expression of DNA Pol-III catalytic core (constituted by the $\alpha \varepsilon \theta$ complex) was attempted. Protein extracts isolated from E. coli TOP10 simultaneously overexpressing $\alpha$, $\varepsilon$, and $\theta$ were subjected to 
gel filtration. Aliquots of each fraction were used to assay exonuclease activity according to Hamdan et al. (2002), and to perform gel electrophoresis. The activity profile features three distinct and sharp peaks (Fig. 2B), which can be ascribed to DNA Pol-III holoenzyme, DNA Pol-III catalytic core, and to the $\varepsilon$ subunit associated with proteins other than $\alpha$ (center of peaks at fractions 6, 23, and 41, respectively). This interpretation is sustained by electrophoretic analyses of the fractions representing the maximum of each activity peak (Fig.s $2 \mathrm{C}$ and 2D). Altogether, these observations indicate that the protein extracts isolated from $E$. coli overexpressing $\alpha, \varepsilon$, and $\theta$ can be used to analyze proteinprotein interactions directly, and suggest an efficient tool for the production and purification of the DNA Pol-III catalytic core.

In addition, by constructing two pGOOD variants (pGOOD1 and pGOOD2), we devised a tightly-controlled expression system. In both pGOOD derivatives, the lacI gene was deleted and replaced with the $\mathrm{lacO}_{i d}$ or $\mathrm{lacO}_{3}$ operator (Oehler et al., 1994), yielding pGOOD1 and pGOOD2, respectively (Fig. 1). The artificial operator $\mathrm{lacO}_{\text {id }}$ features perfect symmetry, and is strongly bound by the LacI repressor. In particular, when a single operator is present in the lac operon, the expression of lacZ is repressed up to $7300,4700,320$, and 16 times in the presence of $\mathrm{lacO}_{i d}, \mathrm{lacO}_{1}, \mathrm{lacO}_{2}$, and $\mathrm{lacO}_{3}$, respectively (Oehler et al., 1994). Moreover, the presence of two operators in the lac operon further increases the extent of repression (Oehler et al., 1994). Accordingly, pGO0D1 (containing the 
lacO $_{i d}-l_{\text {lac }} \mathrm{O}_{1}$ couple, Fig. 1) should sustain very tight repression, while genes cloned in pGOOD2 (containing the $\mathrm{lacO}_{3}$-lacO $\mathrm{I}_{1}$ couple, Fig. 1) should be easier to induce.

To complete our expression system, we inserted the lacI gene or the laclts mutant described by Bukrinsky et al. (1988) into pBAD (Fig. 1). By phenotypic analyses, this temperature-sensitive variant of Lacl was inferred to be stable at $30^{\circ} \mathrm{C}$, and labile at $42^{\circ} \mathrm{C}$ (Bukrinsky at al., 1988). To test our expression system, we inserted the D12A variant of the $\varepsilon$ subunit into pGOOD1. This variant should be devoid of proofreading activity and, accordingly, be responsible for strong mutator phenotypes (Fijalkowska and Schaaper, 1996). In addition, the double mutant $\varepsilon D 12 \mathrm{~A}, \mathrm{E} 14 \mathrm{~A}$ was described as lethal in strains subjected to replacement of the wt dnaQ gene (coding for $\varepsilon$ subunit) with the gene coding for this variant (Fijalkowska and Schaaper, 1996). Accordingly, we reasoned that: i) a tight repression of the gene coding for $\varepsilon D 12 \mathrm{~A}$ would correspond to $E$. coli populations hosting this gene and featuring normal growth rate; ii) the mutation frequency of the host populations would increase under conditions corresponding to instability of Lacl repressor, i.e. under non-permissive temperatures. To test these hypotheses, we co-transformed E. coli TOP10 with: i) pGOOD1 and pBADlacI or pBAD-lacIts; ii) pGOOD1-eD12A and pBAD-lacI or pBAD-lacIts. Each cotransformant was grown at 30 and $42{ }^{\circ} \mathrm{C}$, and the frequency of mutations was determined at each culture temperature. All the co-transformants grew at 
similar rates both at 30 and $42{ }^{\circ} \mathrm{C}$ (data not shown). Interestingly, populations of E. coli TOP10 containing the pGOOD1-عD12A and pBAD-lacI vectors did not feature any significant increase in mutation frequency when grown at $30{ }^{\circ} \mathrm{C}$ and compared with control populations containing the empty pGOOD1 plasmid (Fig. 3). On the contrary, when grown at $42{ }^{\circ} \mathrm{C}$, populations containing pGOOD1$\varepsilon \mathrm{D} 12 \mathrm{~A}$ and pBAD-lacI plasmids mutated at a frequency two to three orders of magnitude higher than the control cultures (Fig. 3). This observation suggests that the LacI repressor is not fully stable at $42{ }^{\circ} \mathrm{C}$ or, alternatively, that at this temperature LacI associates with lac operators with reduced affinity. When the gene coding for the mutagenic $\varepsilon \mathrm{D} 12 \mathrm{~A}$ variant was hosted in cells containing LacI ${ }^{\mathrm{ts}}$, the observed frequency of mutations was higher, both at 30 and $42{ }^{\circ} \mathrm{C}$, than the frequencies detected in control cultures (Fig. 3), suggesting that the LacIts variant described by Bukrinsky et al. (1988) is partially labile at $30^{\circ} \mathrm{C}$.

Altogether, our observations indicate that: i) the expression of extremely toxic proteins in E. coli, such as $\varepsilon \mathrm{D} 12 \mathrm{~A}$, can be tightly controlled using the pGOOD1/pBAD-lacI vector couple; ii) conditional mutators spanning a wide range of frequency of mutations can be obtained using the pG00D1-عD12A plasmid. Remarkably, the desired mutator phenotype, fully conditional or attaining extreme mutation frequencies, can be chosen by exerting repression of $\varepsilon D 12 A$ with LacI or LacIts $($ Fig. 3). 
In conclusion, the two expression systems described herein represent efficient tools for: i) the co-expression of proteins to analyze protein-protein interactions, using the pGOOD-pBAD vector couple; ii) the expression of very toxic proteins in E. coli, using the pGOOD1 and pBAD-lacI plasmids.

\section{Acknowledgements}

We are indebted to Dr. Alberto Danielli (Department of Biology, University of Bologna) for advice and encouragement throughout this work. Thanks are also due to Anne Prudence Collins (Koinè SaS, Editorial Service for Academic Publications) for language editing. Financial support by Mipaaf, Project "Bioenergie" (D.M. n. 337/7303/06, December 21, 2006) is gratefully acknowledged. 


\section{Figure legends}

\section{Figure 1}

Construction of pGOODs. The pTrcHisB plasmid was digested at completion with BstZ17I and AlwNI enzymes; the reaction products were further subjected to partial digestion with the SspI enzyme, and the BstZ17I-SspI(1) fragment was recovered. The pACYC184 vector was totally digested with MscI and BstZ17I enzymes, and the BstZ17I-MscI(1) fragment was recovered. Ligation of the BstZ17I-SspI(1) and BstZ17I-MscI(1) yielded pGOOD, the map of which is indicated according to the orientation determined by restriction analysis. pGOOD was then digested with ApaLI and NsiI enzymes, and the ApaLI(1)-NsiI fragment was recovered. To this fragment, ds-oligonucleotides containing the sequence of $\mathrm{lacO}_{i d}$ or $\mathrm{lacO}_{3}$ operator were ligated, yielding pGOOD1 and pGO0D2, respectively. The resulting distance between $\mathrm{lacO}_{1}$ (residing in the $\mathrm{P} / 0$ element) and the additional operators inserted ( $\mathrm{lacO}_{i d}$ or $\left.\mathrm{lacO}_{3}\right)$ is equal to 200 bp. MCS indicates the location, in the different plasmids, of the multiple cloning site. A map of pBAD containing the lacI gene is also shown. The temperaturesensitive allele of LacI described by Bukrinsky et al. (1988) contains the G187S mutation.

\section{Figure 2}

(A) SDS-PAGE of total proteins extracted from E. coli TOP10 / pBAD- $\alpha 1160$ / pGOOD- $\varepsilon 243$ grown in the absence of inducers, in the presence of arabinose 
only, of IPTG only, or in medium supplemented with both arabinose and IPTG (lanes 1-4, respectively). (B) Absorbance (empty circles) and exonuclease activity (filled circles) of fractions isolated by subjecting to gel-filtration soluble proteins extracted from $E$. coli TOP10 overexpressing $\alpha, \varepsilon$, and $\theta$ subunits of $E$. coli DNA Pol-III. (C) SDS-PAGE of fractions 6, 12, 23, 31, and 41 (lanes 1-5, respectively) reported in panel B. (D) SDS-PAGE of a concentrated aliquot of fraction 23, whose exonuclease activity and electrophoretic pattern are reported in Fig.s 2B and 2C, respectively.

\section{Figure 3}

Frequency of E. coli TOP10 kanamycin-resistant mutants after growth at $30{ }^{\circ} \mathrm{C}$ (black bars) in the presence of pGOOD1 or pGOOD1-عD12A and subjected to overexpression of LacI wt or LacI ${ }^{\mathrm{ts}}$. The frequency of mutants was also determined after further growth at $42{ }^{\circ} \mathrm{C}$ (grey bars) under conditions notinducing LacI repressor. Error bars represent standard deviation ( $\mathrm{n}=3)$. 


\section{References}

Bressanin D, Stefan A, Dal Piaz F, Cianchetta S, Reggiani L, Hochkoeppler A (2009) Proteolysis of the proofreading subunit controls the assembly of Escherichia coli DNA polymerase III catalytic core. Biochim Biophys Acta 179: 1606-1615.

Bukrinsky MI, Barsov EV, Shilov AA (1988) Multicopy expression vector based on temperature-regulated lac repressor: expression of human immunodeficiency virus env gene in Escherichia coli. Gene 70: 415-417.

Fields S, Song O (1989) A novel genetic system to detect protein-protein interactions. Nature 340: 245-246.

Fijalkowska IJ, Schaaper RM (1996) Mutants in the exo motif of Escherichia coli dnaQ: defective proofreading and inviability due to error catastrophe. Proc. Natl. Acad. Sci. USA 93: 2856-2861.

Guzman L, Belin D, Carson M, Beckwith J (1995) Tight regulation, modulation, and high-level expression by vectors containing the arabinose $\mathrm{P}_{\mathrm{BAD}}$ promoter. J. Bacteriol. 177: 4121-4130. 
Ha T, Enderle T, Ogletree DF, Chemla DS, Selvin PR, Weiss S (1996) Probing the interaction between two single molecules: fluorescence resonance energy transfer between a single donor and a single acceptor. Proc. Natl. Acad. Sci. USA 93: 6264-6268.

Hamdan S, Bulloch EM, Thompson PR, Beck JL, Yang JY, Crowther JA, Lilley PE, Carr PD, Ollis DL, Brown SE, Dixon NE (2002) Hydrolysis of the 5'-p-nitrophenyl ester of TMP by the proofreading exonuclease (epsilon) subunit of Escherichia coli DNA polymerase III. Biochemistry 41: 5266-5275.

Joung JK, Ramm EI, Pabo CO (2000) A bacterial two-hybrid selection system for studying protein-DNA and protein-protein interactions. Proc. Natl. Acad. Sci. USA 97: 7382-7387.

Karimova G, Pidoux J, Ullmann A, Ladant D (1998) A bacterial two-hybrid system based on a reconstituted signal transduction pathway. Proc. Natl. Acad. Sci. USA 95: 5752-5756.

Lehming N, Sartorius J, Niemöller M, Genenger G, von Wilcken-Bergmann B, Müller-Hill B (1987) The interaction of the recognition helix of lac repressor with lac operator. EMBO J. 6: 3145-3153. 
Lenski RE, Bouma JE (1987) Effects of segregation and selection on instability of plasmid pACYC184 in Escherichia coli. J. Bacteriol. 169: 5314-5316.

Monteiro ACM, Ferreira RCC, Padilla G, Ferreira LCS, Costa SOP (2003) Environmental and genetic factors affecting mutability to aminoglycoside antibiotics among Escherichia coli K12 strains. Genetics and Mol. Biol. 26: 221227.

Oehler S, Amouyal M, Kolkhof P, von Wicken-Bergmann B, Müller-Hill B (1994) Quality and position of the three lac operators of E. coli define efficiency of repression. EMBO J. 13: 3348-3355.

Sambrook J, Russell DW (2001) Molecular Cloning: A Laboratory Manual, 3rd edn. Cold Spring Harbor (New York): Cold Spring Harbor Laboratory Press.

Stryer L, Haugland RP (1967) Energy transfer: a spectroscopic ruler. Proc. Natl. Acad. Sci. USA 58: 719-726.

Walia R, Deb JK, Mukherjee KJ (2007) Development of expression vectors for Escherichia coli based on the pCR2 replicon. Microb. Cell Fact. 6: 14. 
Zeng J, Zhang L, Li Y, Wang Y, Wang M, Duan X, He Z (2010) Over-producing soluble protein complex and validating protein-protein interaction through a new bacterial co-expression system. Protein Express. Purif. 69: 47-53.

Zhang H, Wu H, Zhang H (2007) A novel high-copy plasmid, pEC, compatible with commonly used Escherichia coli cloning and expression vectors. Biotechnol. Lett. 29: 431-437.
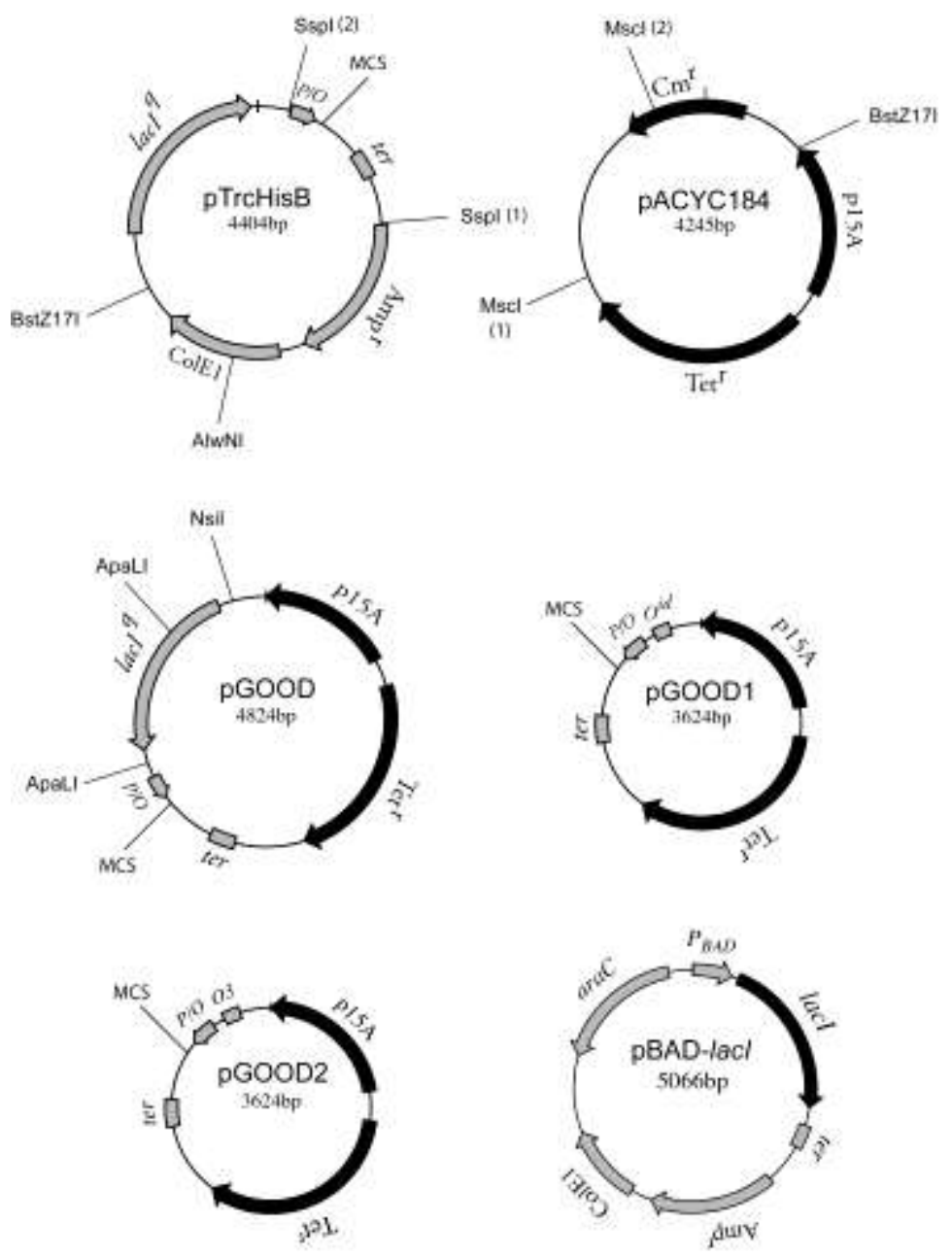

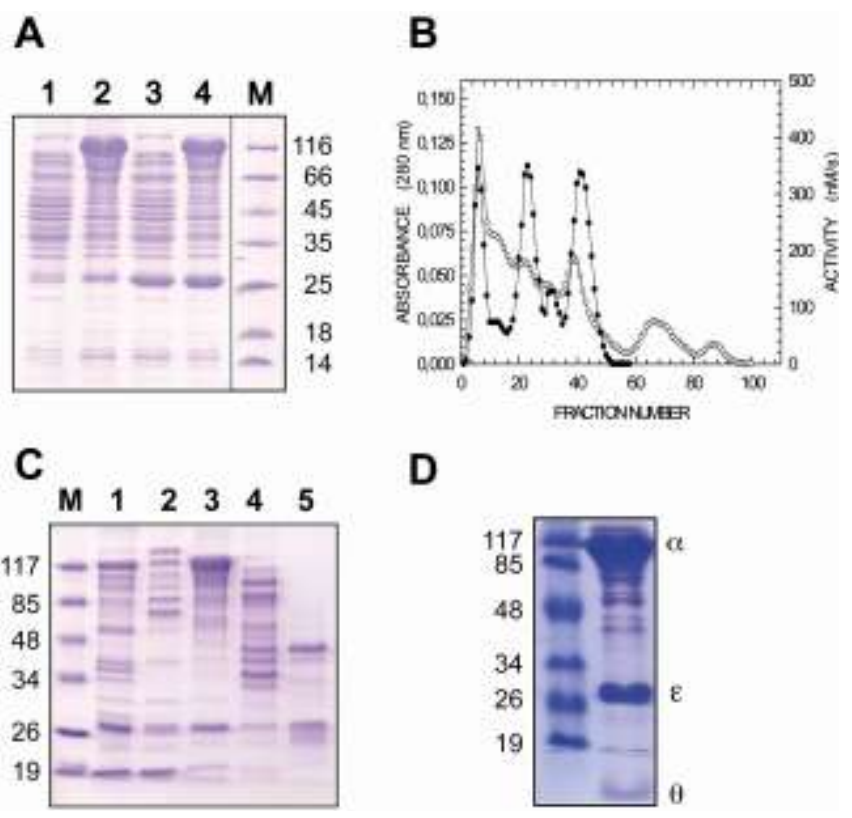

D
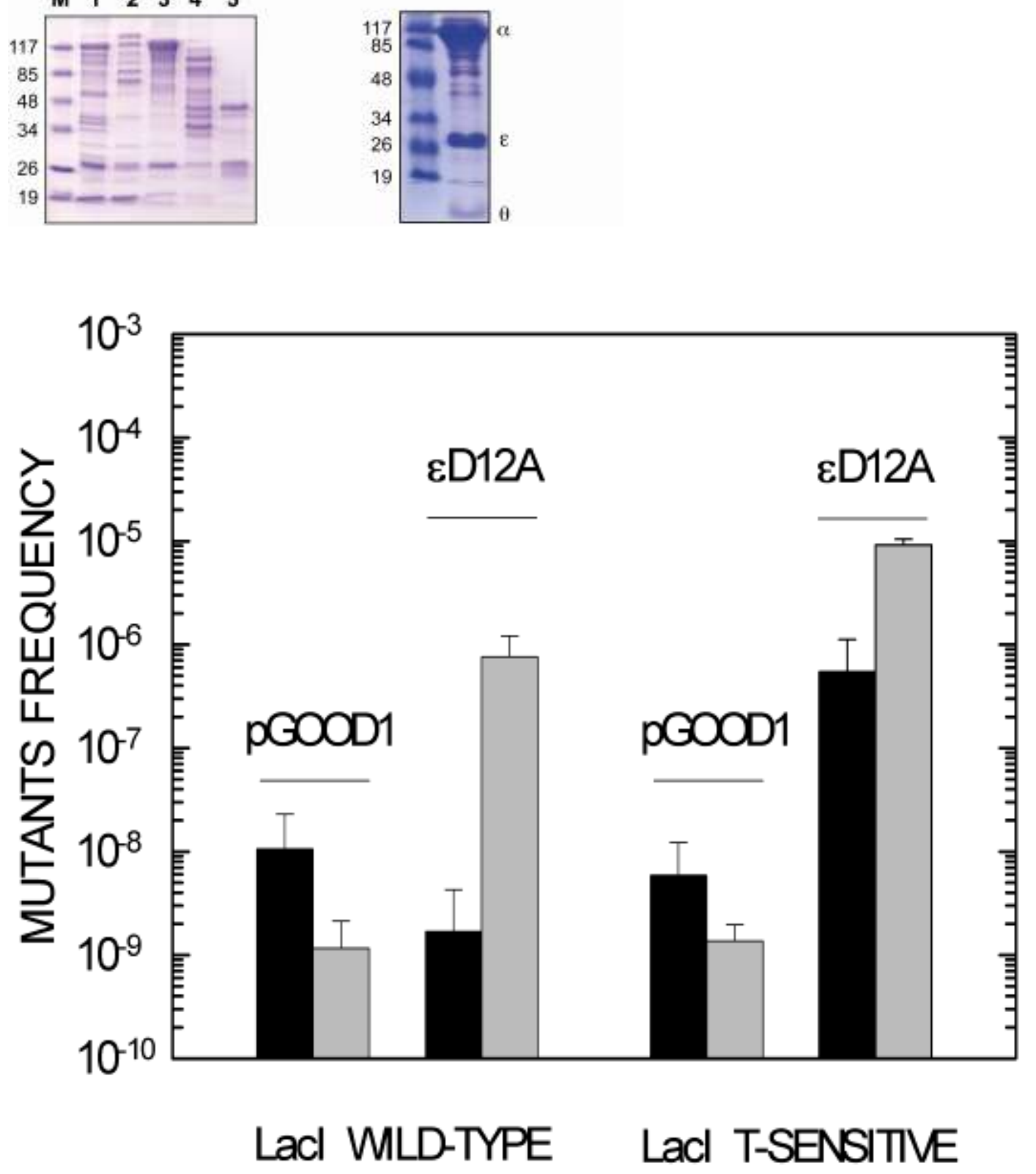